\title{
CHARACTERIZATION OF FIBER MISALIGNMENT BY COMPUTED TOMOGRAPHY COUPLED WITH DIGITAL IMAGE CORRELATION
}

\author{
Mahoor Mehdikhani", Christian Breite, Yentl Swolfs, Larissa Gorbatikh, Martine Wevers, \\ Stepan V. Lomov
}

KU Leuven, Department of Materials Engineering, Kasteelpark Arenberg 44, 3001, Leuven, Belgium

*mahoor.mehdikhani@kuleuven.be

A methodology is developed to employ Digital Image Correlation (DIC) as a potential technique for post-processing micro-CT volumes to measure the misalignment of the fibers. This is a special application of DIC since DIC is applied to a 3D image taken at a single time step to quantify material structure, while typically it is applied to a set of 2D images taken over time to quantify the deformation. By tracking the fiber cross-sections in each CT slice, DIC can quantify the location of each fiber through the whole volume (Fig. 1). The change in fiber locations through consecutive slices is then translated to in-plane and out-of-plane orientations.

The methodology is applied to synchrotron CT images of a glass/epoxy laminate with a voxel size of $1.1 \mu \mathrm{m}$. It is found that the fibers are almost straight. Thus, their orientation is calculated with linear fits to their location. Statistical tests show an excellent agreement between the DIC-based orientation distributions and those obtained via VoxTex [1]. It is found that the average/standard deviation of the DIC displacement field in every CT slice is a good estimation of the average/standard deviation of the actual displacement of all fibers' cross-sections in that slice. This provides a shortcut to estimate the fiber orientation distribution, compared to the algorithms that reconstruct the fiber center lines. Using this shortcut, the fiber misalignment was also characterized in synchrotron CT images of a carbon/epoxy laminate, where individual fibers were not clearly observed and where, fiber segmentation tools failed to characterize the fibers. The resulting orientation distribution is found to match well with the VoxTex results. Additionally, the evolution of the fiber misalignment during tensile loading is investigated. The local misalignment analysis is performed on a set of synchrotron volumes acquired in-situ during loading. Negligible changes of misalignment with tensile loading have been noted.

The proposed methodology is objective since it does not need any training of the algorithm. Furthermore, it can be used by anyone who has access to DIC software. As a drawback, DIC subset averaging can lead to an underestimation of the misalignments. This can be diminished by using smaller subsets, of course up to the point that the subsets still contain enough grayscale variability to build recognizable patterns.
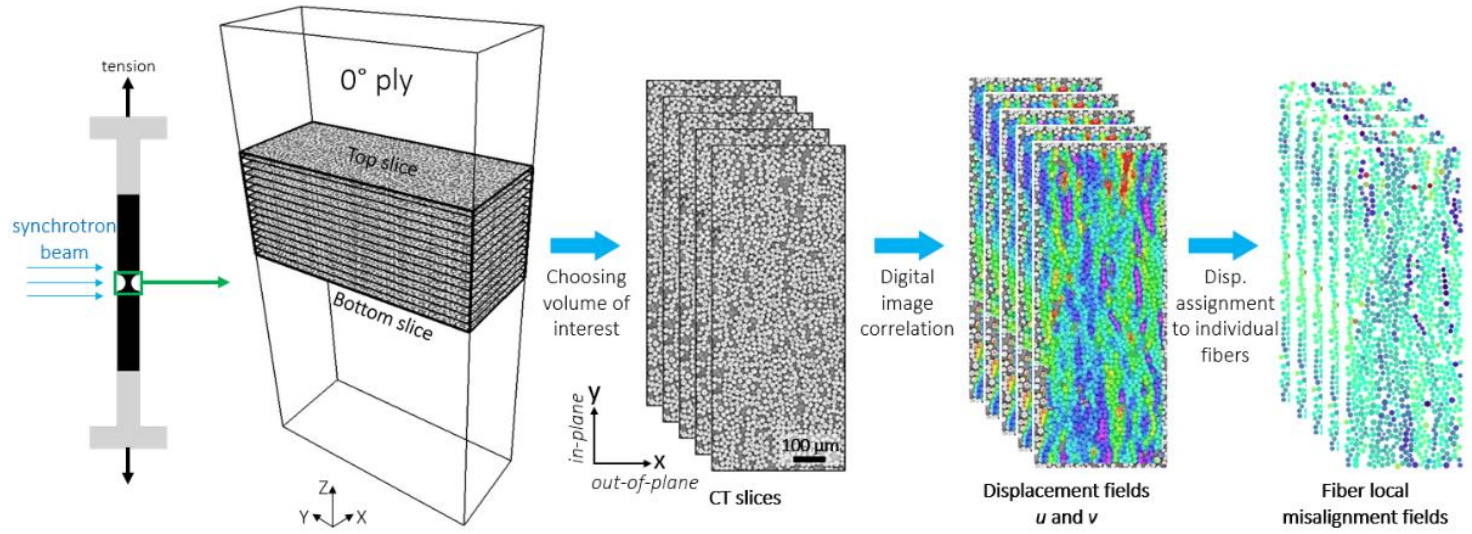

Figure 1. Roadmap of DIC misalignment measurement methodology, starting from image acquisition.

\section{References}

[1] I. Straumit, S.V. Lomov, M. Wevers (2015) Quantification of the internal structure and automatic generation of voxel models of textile composites from X-ray computed tomography data. Composites: Part A, 69, 150.

\section{Acknowledgments}

The study is funded by the KU Leuven Research Council, project C24/17/052 and an FWO Postdoc Fellowship, project ToughImage $(1263421 \mathrm{~N})$. C. Breite acknowledges the FiBreMoD project, which has received funding from the European Union's Horizon 2020 research and innovation programme under the Marie Skłodowska-Curie grant agreement No 722626. The authors acknowledge the Paul Scherrer Institut, Villigen, Switzerland for provision of synchrotron radiation beamtime at the beamline TOMCAT of the SLS, proposal No. 20161157. 